Gloucestershire outpatient departments. A spreadsheet with datafields for postcode, diagnoses and SNOMED mappings within the BSG dataset was developed. Microsoft MapPoint ${ }^{\circledR}$ was used to examine the geographic distribution of the cohort with the intention of improving follow-up arrangements for patients in relation to the location of community hospitals.

Results From 236 patients studied to date, 100\% of patients could be assigned a code from within the dataset. However, $32.2 \%$ had alternative/co-existing diagnoses that could also be legitimately coded. In order to be clinically useful the outpatient record should contain datafields for both aetiology (specific) and stage of liver disease (generic). For example, the MapPoint exercise provided an insightful distinction between requirements for a viral hepatitis or cirrhosis clinic in community hospitals.

Conclusion The BSG hepatology dataset satisfies the scoping requirements of HSCIC but a single diagnostic datafield entry is not immediately useful to clinicians, service providers or commissioners since treatment pathways in terms of aetiology and management pathways in terms of stage need not correlate. Disclosure of Interest None Declared.

\section{PWE-138 SEPSIS INDUCED LIVER DYSFUNCTION: EARLY DIAGNOSTIC AND PROGNOSTIC MARKERS - THE SINGLETON EXPERIENCE}

H Glover*, M Sherlock, CL Ch'ng. Gastroenterology, ABMU Health Board, Singleton Hospital, Swansea, UK

\subsection{6/gutjnl-2014-307263.398}

Introduction To evaluate the incidence of liver dysfunction in septic patients and to determine the best available biomarker widely available as a diagnostic and prognostic marker of mortality.

Methods Adult inpatients (aged over 18) with positive blood cultures were identified from the microbiology database between Jan 2012 and June 2012. Total protein, albumin, bilirubin, ALP and ALT were recorded pre, peri and post sepsis. Peak derangement of liver function test (LFT) was evaluated. $\mathrm{Hb}$, WCC, Plt and CRP were recorded on the date of positive blood culture. Patients fell into 3 groups; normal liver function, alcoholic liver disease and non-alcoholic liver disease. Kaplan Meier survivorship scores and ROC curves were calculated in SPSS ${ }^{\mathrm{R}}$.

Results 93 of 140 patients with positive blood cultures had abnormal LFTs during admission. 71 medical case records were available for review. 41 patients had normal LFTs prior to admission, 30 had pre-existing liver disease with abnormal LFTs (8 ALD; 22 with malignancy). The median age of the cohort was 66.7 yrs (23-93) with an equal sex distribution (35 M:37 F). 47/ 71 patients had deranged LFTs prior to documented bacteraemia, 19/71 on the day of bacteraemia and 5/71 after.

Bilirubin was the most sensitive parameter of the LFT in predicting mortality prior to organism culture,calculated using ROC curves with an area of 0.59 . Following positive blood culture, bilirubin, ALT and CRP rises are indicators of mortality with areas of $0.64,0.55$ and 0.55 respectively. The ROC curves were not statistically significant for $\mathrm{Hb}$, WCC and platelets prior to,or after the onset of bacteraemia.

4 patients died within $24 \mathrm{~h}, 4$ between $24-72 \mathrm{~h}$ and 7 between $72 \mathrm{~h}$ and 30 days. The overall mortality was 30\% lower than a comparative study at $44.7 \%$ (median age 66.7 ). ${ }^{1}$ There was no statistically significant difference in mortality from sepsis with pre-existing liver disease (alcoholic or malignant) compared to no existing liver disease.

Sepsis-induced liver dysfunction was present on admission in $66 \%$ of septic patients with previously normal LFT's, $27 \%$ prior to positive blood cultures and $5 \%$ after positive blood cultures. This is comparative to an incidence at admission of $58.3 \%$ in a recent study. ${ }^{1}$ The relative risk of mortality in the presence of sepsis induced liver injury was 1.82 .

Conclusion Sepsis-induced liver dysfunction is common and clinically important to identify and has prognostic implications. Abnormal liver function can precede organism culture. There is currently no widely available gold standard test reflecting liver failure. Bilirubin is a diagnostic and prognostic marker of mortality before and after the onset of sepsis. ALT and CRP are useful after the onset of sepsis.

\section{REFERENCE}

1 Kobashi et al. Sepsis associated liver injury:Incidence,classification and the clinical significance. Hepatology Research 2013;43:255-266

Disclosure of Interest None Declared.

\section{PWE-139 TACE IN THE MANAGEMENT OF HCC IN A REGIONAL CENTRE: 5 YEAR ANALYSIS AND ASSESSMENT OF PREDICTORS OF OUTCOME}

${ }^{1}$ ID Morrison*, ${ }^{2} \mathrm{R}$ Kasthuri, ${ }^{1}$ EH Forrest, ${ }^{1} \mathrm{~S}$ Barclay, ${ }^{1} \mathrm{R}$ Gillespie, ${ }^{3} \mathrm{PR}$ Mills, ${ }^{3} \mathrm{M}$ Priest, 4J Evans, ${ }^{1}$ AJ Stanley. 'Gastroenterology, Glasgow Royal Infirmary, Glasgow, UK; ${ }^{2}$ Interventional Radiology, Glasgow, UK; ${ }^{3}$ Gastroenterology, Gartnavel General Hospital, Glasgow, UK; ${ }^{4}$ Medical Oncology, Beatson Oncology Centre, Glasgow, UK

\subsection{6/gutjnl-2014-307263.399}

Introduction Transarterial chemoembolisation (TACE) is a useful treatment for selected patients unsuitable for surgical management of hepatocellular carcinoma (HCC). The Hepatoma Arterial-embolization Prognostic (HAP) score has been proposed to be a a better predictor of post-TACE outcome than the ChildPugh or BCLC (Barcelona clinic liver cancer) scores. ${ }^{1}$

Methods Patients diagnosed with HCC from January 2008 until December 2012 were identified from a prospectively compiled regional MDT database. Patients were risk stratified by Child Pugh grade, BCLC and HAP scores. Response to treatment was assessed by the mRECIST criteria (modified response evaluation criteria in solid tumours). ${ }^{2}$ Relationship between risk scores and outcomes were assessed using Log-Rank tests and median survivals.

Results 282 patients were diagnosed with HCC during the study period. 101 of these patients (81 male, 20 female) mean age 66.0 (SD 10.1 years, range 37 to 85 ) were treated locally with TACE. Aetiology was alcoholic liver disease in 30\%, unknown in $21 \%$, non alcoholic liver disease $15 \%$, viral hepatitis $12 \%$, haemochromatosis $8 \%$, other and mixed aetiology 14\%. Baseline Child-Pugh grades A, B and C were 76, 21 and 3\% respectively. BCLC Staging was A, B, C and D in 25, 58, 13 and 4\% respectively. HAP Scores A, B, C and D were 14, 39, 37 and 11\% respectively.

A total of 228 TACE procedures were performed (mean 2.3 per patient; range 1-6). In $10(10 \%)$ of patients, TACE was used in combination with radiofrequency ablation and in two $(2 \%)$ cases it was successfully used as a bridge to transplant. $88 \%$ of patients had TACE as sole therapy. Radiological followup post TACE was performed in 208 occasions with $18 \%$ having a mRECIST complete response, $43 \%$ a partial response, $26 \%$ static disease and $14 \%$ progressive disease. 